\title{
Relaxation of Vibrationally Excited OH Radical by SO Supporting Information
}

\author{
Juan de Dios Garrido, Samah Ellakkis and Maikel Y. Ballester * \\ E-mail: maikel.ballester@ufjf.edu.br
}
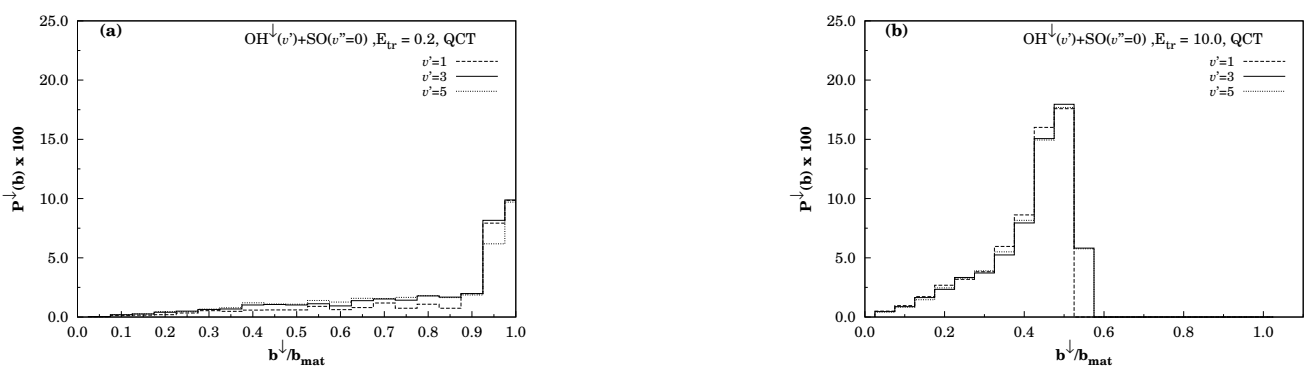

Figure S1: Opacity functions for $E_{t r}=0.2 \mathrm{kcal} \mathrm{mol}^{-1}$ (panel (a)) and $E_{t r}=10.0 \mathrm{kcal} \mathrm{mol}^{-1}$ (panel (b)). In both panels appear graphics for excited vibrational states of the $\mathrm{OH}$ corresponding to the vibrational quantum numbers $v^{\prime}=1,3,5$

Table S1: Numerical values for the coefficients in the functions $f^{\mathrm{OH}}\left(E_{i}\right)$ and $g^{\mathrm{OH}}\left(E_{i}\right)$ for the initial state deactivation cross section corresponding to the QCT approach.

\begin{tabular}{cccc}
\hline$c_{0}$ & $c_{1}$ & $c_{2}$ & $c_{3}$ \\
-2.01097 & 0.351752 & 0.00987273 & -0.000184503 \\
$d_{0}$ & $d_{1}$ & $d_{2}$ & $d_{3}$ \\
0.36019 & 0.0202692 & 0.00612129 & $-5.66337 \mathrm{e}-05$ \\
\hline
\end{tabular}




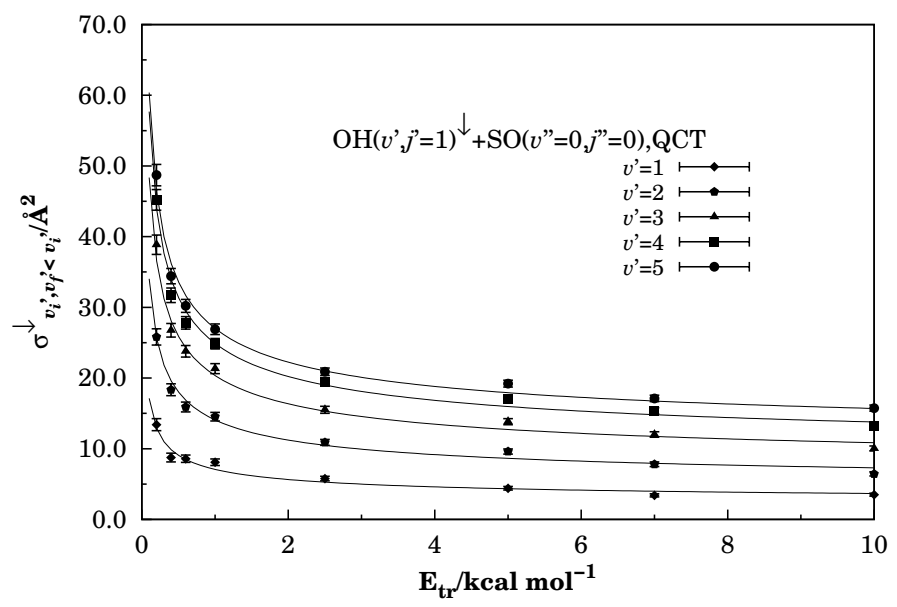

Figure S2: Specific initial state deactivation cross section for the non-reactive collisions $\mathrm{OH}\left(v^{\prime}, j^{\prime}=1\right)+\mathrm{SO}\left(v^{\prime \prime}=0, j^{\prime \prime}=0\right)$ for the QCT approximation.

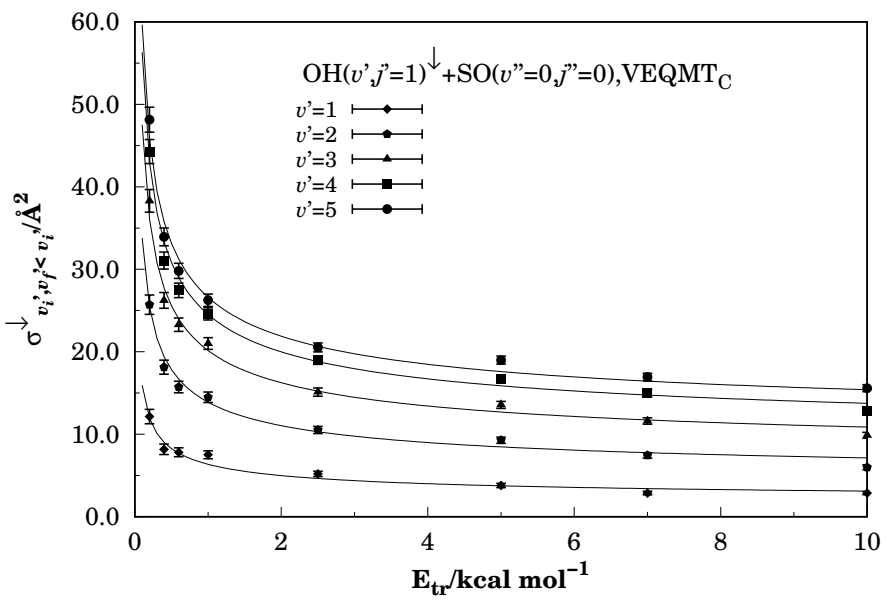

Figure S3: Specific initial state deactivation cross section for the non-reactive collisions $\mathrm{OH}\left(v^{\prime}, j^{\prime}=1\right)+\mathrm{SO}\left(v^{\prime \prime}=0, j^{\prime \prime}=0\right)$ in the VEQMTc approach. 
Table S2: Numerical values for the coefficients in the functions $f^{\mathrm{OH}}\left(E_{i}\right)$ and $g^{\mathrm{OH}}\left(E_{i}\right)$ for the initial state deactivation cross section corresponding to the VEQMTc approach.

\begin{tabular}{cccc}
\hline$c_{0}$ & $c_{1}$ & $c_{2}$ & $c_{3}$ \\
-5.32077 & 0.705464 & -0.00202971 & -0.0000638684 \\
$d_{0}$ & $d_{1}$ & $d_{2}$ & $d_{3}$ \\
-0.0272548 & -0.0293596 & 0.00969124 & -0.000106071 \\
\hline
\end{tabular}
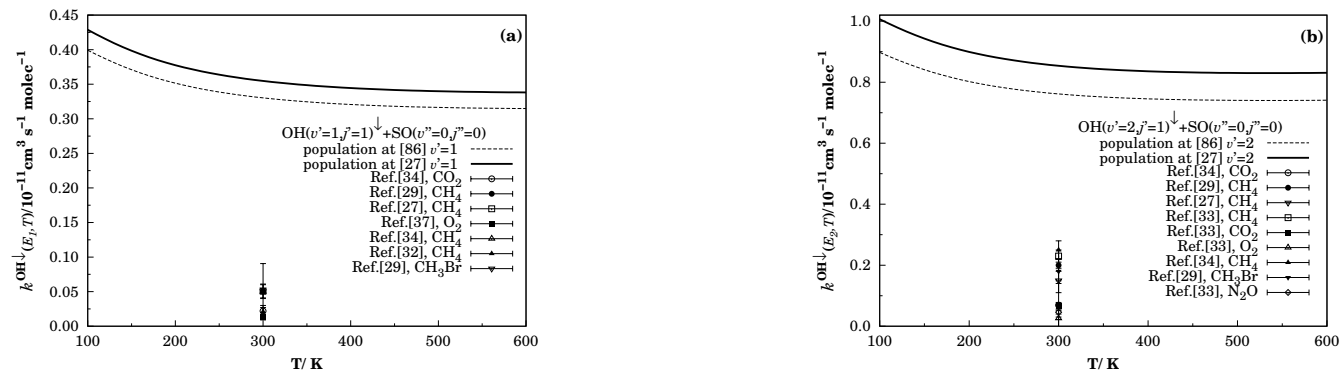

Figure S4: Specific initial state deactivation thermal rate coefficients for $\mathrm{OH}\left(v^{\prime}, 1\right)$, with $v^{\prime}=1,2,3$, compared with experimental data for deactivation of vibrationally excited $\mathrm{OH}$ by non-effective relaxants.
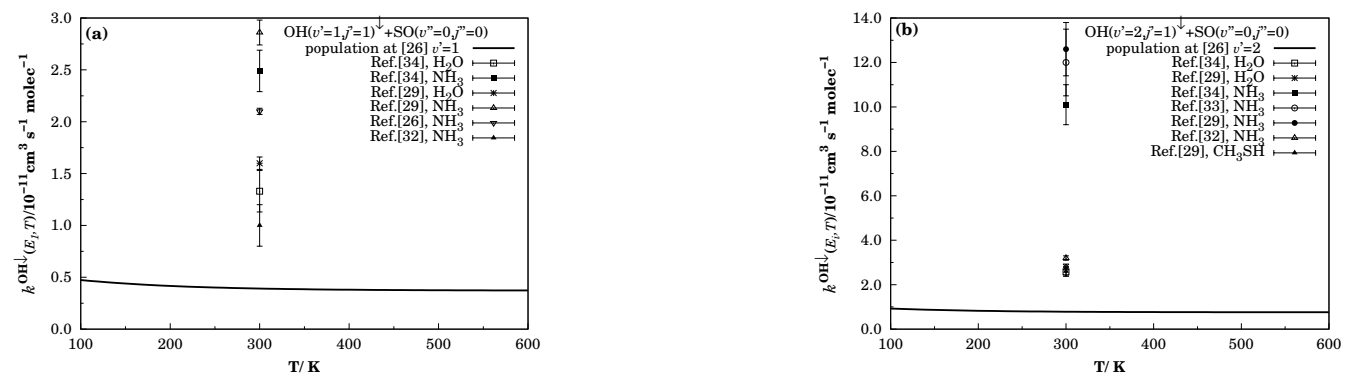

Figure S5: Specific initial state deactivation thermal rate coefficients for $\mathrm{OH}\left(v^{\prime}, 1\right)$, with $v^{\prime}=1,2,3$, compared with experimental data for deactivation of vibrationally excited $\mathrm{OH}$ by effective relaxants. 
Table S3: Summary of the trajectory calculations for the collisions $\mathrm{OH}\left(v_{i}^{\prime}, j_{i}^{\prime}=1\right)+\mathrm{SO}$ $\left(v_{i}^{\prime \prime}=0, j_{i}^{\prime \prime}=0\right)$. The third to fifth columns collect results for the QCT calculations. The columns numbers 6, 7 and 8 show the VEQMTc approach. SCATT. indicates the number of scattered trajectories. COMPL. denote the number of trajectories leading to complex formation.

\begin{tabular}{|c|c|c|c|c|c|c|c|}
\hline$v^{\prime}$ & $E_{t r}$ & QCT & SCATT. & COMPL. & VEQMTc & SCATT. & COMPL. \\
\hline \multirow[t]{8}{*}{1} & 0.2 & 4995 & 1471 & 3524 & 4343 & 1421 & 2922 \\
\hline & 0.4 & 4990 & 1659 & 3331 & 4368 & 1623 & 2745 \\
\hline & 0.6 & 4996 & 1385 & 3611 & 4179 & 1324 & 2855 \\
\hline & 1.0 & 4999 & 1735 & 3264 & 4315 & 1678 & 2637 \\
\hline & 2.5 & 4999 & 2139 & 2860 & 4338 & 2072 & 2266 \\
\hline & 5.0 & 4990 & 2778 & 2212 & 4469 & 2716 & 1753 \\
\hline & 7.0 & 4997 & 3018 & 1979 & 4520 & 2966 & 1554 \\
\hline & 10.0 & 4998 & 3051 & 1947 & 4581 & 2964 & 1617 \\
\hline \multirow[t]{8}{*}{2} & 0.2 & 4997 & 1701 & 3296 & 4786 & 1680 & 3106 \\
\hline & 0.4 & 4996 & 1653 & 3343 & 4754 & 1627 & 3127 \\
\hline & 0.6 & 5000 & 1514 & 3486 & 4740 & 1484 & 3256 \\
\hline & 1.0 & 4997 & 1993 & 3004 & 4753 & 1967 & 2786 \\
\hline & 2.5 & 4999 & 2213 & 2786 & 4772 & 2168 & 2604 \\
\hline & 5.0 & 5000 & 2815 & 2185 & 4801 & 2772 & 2029 \\
\hline & 7.0 & 4998 & 3017 & 1981 & 4822 & 2978 & 1844 \\
\hline & 10.0 & 5000 & 3136 & 1864 & 4841 & 3092 & 1749 \\
\hline \multirow[t]{8}{*}{3} & 0.2 & 4998 & 1784 & 3214 & 4916 & 1763 & 3153 \\
\hline & 0.4 & 4998 & 1564 & 3434 & 4914 & 1540 & 3374 \\
\hline & 0.6 & 4999 & 1539 & 3460 & 4911 & 1511 & 3400 \\
\hline & 1.0 & 4992 & 1984 & 3008 & 4910 & 1960 & 2950 \\
\hline & 2.5 & 4998 & 2365 & 2633 & 4890 & 2328 & 2562 \\
\hline & 5.0 & 4999 & 2814 & 2185 & 4906 & 2782 & 2124 \\
\hline & 7.0 & 4998 & 3013 & 1985 & 4910 & 2974 & 1936 \\
\hline & 10.0 & 5000 & 3220 & 1780 & 4917 & 3198 & 1719 \\
\hline \multirow[t]{8}{*}{4} & 0.2 & 4999 & 1822 & 3177 & 4962 & 1800 & 3162 \\
\hline & 0.4 & 4997 & 1606 & 3391 & 4959 & 1584 & 3375 \\
\hline & 0.6 & 4998 & 1661 & 3337 & 4961 & 1644 & 3317 \\
\hline & 1.0 & 4999 & 1910 & 3089 & 4963 & 1894 & 3069 \\
\hline & 2.5 & 4997 & 2205 & 2792 & 4943 & 2172 & 2771 \\
\hline & 5.0 & 4999 & 2900 & 2099 & 4951 & 2876 & 2075 \\
\hline & 7.0 & 4996 & 3018 & 1978 & 4942 & 2984 & 1958 \\
\hline & 10.0 & 4999 & 3203 & 1796 & 4939 & 3168 & 1771 \\
\hline
\end{tabular}


Table S4: Summary of the trajectory calculations for the collisions $\mathrm{OH}\left(v_{i}^{\prime}, j_{i}^{\prime}=1\right)+\mathrm{SO}$ $\left(v_{i}^{\prime \prime}=0, j_{i}^{\prime \prime}=0\right)$. The third to fifth columns collect results for the QCT calculations. The columns numbers 6, 7 and 8 show the VEQMTc approach. SCATT. indicates the number of scattered trajectories. COMPL. denote the number of trajectories leading to complex formation.(continuation)

\begin{tabular}{cccccccc}
\hline (continuation) & & & & & & \\
$v^{\prime}$ & $E_{t r}$ & QCT & SCATT. & COMPL. & VEQMTc & SCATT. & COMPL. \\
5 & 0.2 & 5000 & 1824 & 3176 & 4977 & 1810 & 3167 \\
& 0.4 & 4999 & 1725 & 3274 & 4976 & 1709 & 3267 \\
& 0.6 & 4999 & 1787 & 3212 & 4975 & 1771 & 3204 \\
& 1.0 & 5000 & 1969 & 3031 & 4983 & 1961 & 3022 \\
& 2.5 & 5000 & 2253 & 2747 & 4958 & 2224 & 2734 \\
& 5.0 & 5000 & 2876 & 2124 & 4975 & 2857 & 2118 \\
& 7.0 & 4999 & 3068 & 1931 & 4974 & 3051 & 1923 \\
& 10.0 & 4998 & 3220 & 1778 & 4972 & 3204 & 1768 \\
\hline
\end{tabular}

Table S5: Electrostatic dipole and quadrupole moments and polarizabilities for some selected molecular species.(T) stands for theoretical calculation.

\begin{tabular}{cccc}
\hline Molecule & Dipole moment $(\mathrm{D})$ & Quadrupole moment $(\mathrm{DA})$ & Polarizability $\left(\AA^{3}\right)$ \\
$\mathrm{OH}$ & $1.639^{a}(\mathrm{~T})$ & - & - \\
$\mathrm{OH}$ & $1.66^{b, c}$ & $-1.610^{b}(\mathrm{~T})$ & $0.722^{b}(\mathrm{~T})$ \\
$\mathrm{SO}$ & $1.207^{a}(\mathrm{~T})$ & $-1.345^{a}(\mathrm{~T})$ & - \\
$\mathrm{SO}$ & $1.55^{b}$ & $-1.26433^{b}(\mathrm{~T})$ & $3.235^{b}(\mathrm{~T})$ \\
$\mathrm{O}_{2}$ & 0 & $-0.310^{b}$ & $1.562^{d}$ \\
$\mathrm{CH}_{4}$ & 0 & 0 & $2.448^{d}$ \\
$\mathrm{H}_{2} \mathrm{O}$ & $1.85^{b, c}$ & $-0.13^{b}$ & $1.501^{d}$ \\
$\mathrm{H}_{2} \mathrm{O}$ & $1.85498^{e}$ & - & $2.103^{d}$ \\
$\mathrm{NH}_{3}$ & $1.47^{b, c}$ & $-2.32^{b}$ & - \\
\hline
\end{tabular}

${ }^{a}$ Values from the $\mathrm{HSO}_{2}$ DMBE PES [59]. ${ }^{b}$ NIST Computational Chemistry Comparison and Benchmark Database NIST Standard Reference Database. http://cccbdb.nist.gov/.

${ }^{c}$ Ralph D. Nelson, Jr., David R. Lide Jr, and Arthur A. Maryott Selected Values of Electric Dipole Moments for Molecules in the Gas Phase, National Standard Reference

Data Series-National Bureau of Standards 10, (Category 3 Atomic and Molecular Properties). Issued September 1, 1967. ${ }^{d}$ Terry N. Olney, N.M. Cann, Glyn Cooper, C.E. Brion, Chem. Phys. 223,59 (1997). e S.L. Shostak, W.L. Ebenstein, J.S. Muenter, J. Chem. Phys., 94, 5875 (1991). 
Table S6: State-to-state deactivation probability for $\mathrm{OH}\left(v^{\prime}, j^{\prime}=1\right)+\mathrm{SO}\left(v^{\prime \prime}=0, j^{\prime \prime}=0\right)$ collisions. IVEQMT approximation.

\begin{tabular}{|c|c|c|c|c|c|c|}
\hline change & $E_{t r}$ & $\mathrm{OH}(5,1)$ & $\mathrm{OH}(4,1)$ & $\mathrm{OH}(3,1)$ & $\mathrm{OH}(2,1)$ & $\mathrm{OH}(1,1)$ \\
\hline \multirow[t]{8}{*}{$v^{\prime} \rightarrow v^{\prime}-1$} & 0.2 & 0.051 & 0.065 & 0.082 & 0.069 & 0.044 \\
\hline & 0.4 & 0.042 & 0.065 & 0.076 & 0.066 & 0.041 \\
\hline & 0.6 & 0.046 & 0.062 & 0.083 & 0.072 & 0.052 \\
\hline & 1.0 & 0.059 & 0.071 & 0.089 & 0.078 & 0.059 \\
\hline & 2.5 & 0.066 & 0.085 & 0.086 & 0.086 & 0.060 \\
\hline & 5.0 & 0.072 & 0.082 & 0.092 & 0.089 & 0.048 \\
\hline & 7.0 & 0.072 & 0.079 & 0.076 & 0.069 & 0.039 \\
\hline & 10.0 & 0.064 & 0.068 & 0.063 & 0.057 & 0.041 \\
\hline \multirow[t]{8}{*}{$v^{\prime} \rightarrow v^{\prime}-2$} & 0.2 & 0.043 & 0.043 & 0.031 & 0.018 & \\
\hline & 0.4 & 0.041 & 0.043 & 0.036 & 0.018 & \\
\hline & 0.6 & 0.044 & 0.048 & 0.037 & 0.020 & \\
\hline & 1.0 & 0.049 & 0.054 & 0.035 & 0.019 & \\
\hline & 2.5 & 0.057 & 0.055 & 0.043 & 0.021 & \\
\hline & 5.0 & 0.053 & 0.046 & 0.045 & 0.020 & \\
\hline & 7.0 & 0.047 & 0.045 & 0.043 & 0.017 & \\
\hline & 10.0 & 0.042 & 0.041 & 0.034 & 0.021 & \\
\hline \multirow[t]{8}{*}{$v^{\prime} \rightarrow v^{\prime}-3$} & 0.2 & 0.030 & 0.025 & 0.011 & & \\
\hline & 0.4 & 0.030 & 0.022 & 0.010 & & \\
\hline & 0.6 & 0.031 & 0.026 & 0.013 & & \\
\hline & 1.0 & 0.033 & 0.024 & 0.013 & & \\
\hline & 2.5 & 0.036 & 0.030 & 0.013 & & \\
\hline & 5.0 & 0.038 & 0.029 & 0.014 & & \\
\hline & 7.0 & 0.035 & 0.030 & 0.018 & & \\
\hline & 10.0 & 0.032 & 0.029 & 0.019 & & \\
\hline \multirow[t]{8}{*}{$v^{\prime} \rightarrow v^{\prime}-4$} & 0.2 & 0.017 & 0.009 & & & \\
\hline & 0.4 & 0.020 & 0.008 & & & \\
\hline & 0.6 & 0.020 & 0.008 & & & \\
\hline & 1.0 & 0.024 & 0.011 & & & \\
\hline & 2.5 & 0.026 & 0.012 & & & \\
\hline & 5.0 & 0.028 & 0.011 & & & \\
\hline & 7.0 & 0.026 & 0.017 & & & \\
\hline & 10.0 & 0.025 & 0.017 & & & \\
\hline
\end{tabular}


Table S7: Initial-state deactivation probabilities for the IVEQMT approach

\begin{tabular}{cccccc}
\hline$E_{t r}$ & $E_{i}$ & Prob & $E_{t r}$ & $E_{i}$ & Prob \\
0.2 & 15.5247 & 0.0439 & 0.2 & 25.2699 & 0.0873 \\
0.4 & & 0.0409 & 0.4 & & 0.0839 \\
0.6 & & 0.0519 & 0.6 & & 0.0921 \\
1.0 & & 0.0595 & 1.0 & & 0.0970 \\
2.5 & & 0.0598 & 2.5 & & 0.1068 \\
5.0 & & 0.0485 & 5.0 & & 0.1096 \\
7.0 & & 0.0391 & 7.0 & & 0.0860 \\
10.0 & & 0.0408 & 10.0 & & 0.0782 \\
0.2 & 34.5578 & 0.1246 & 0.2 & 43.3830 & 0.1419 \\
0.4 & & 0.1222 & 0.4 & & 0.1385 \\
0.6 & & 0.1327 & 0.6 & & 0.1445 \\
1.0 & & 0.1367 & 1.0 & & 0.1605 \\
2.5 & & 0.1416 & 2.5 & & 0.1813 \\
5.0 & & 0.1505 & 5.0 & & 0.1694 \\
7.0 & & 0.1361 & 7.0 & & 0.1725 \\
10.0 & & 0.1163 & 10.0 & & 0.1544 \\
0.2 & 51.7369 & 0.1482 & - & - & - \\
0.4 & & 0.1410 & - & & - \\
0.6 & & 0.1529 & - & & - \\
1.0 & & 0.1734 & - & & - \\
2.5 & & 0.1953 & - & & - \\
5.0 & & 0.2011 & - & & - \\
7.0 & & 0.1893 & - & & - \\
10.0 & & 0.1780 & - & & - \\
\hline
\end{tabular}

Table S8: Experimental initial state deactivation rate coefficients for the $\mathrm{OH}$ relaxation by relaxants with intermediate effectiveness $\mathrm{CH}_{4}, \mathrm{CO}_{2}, \mathrm{~N}_{2} \mathrm{O}, \mathrm{O}_{2}$. Values are given in $10^{-12} \mathrm{~cm}^{3} \mathrm{~s}^{-1}$.

\begin{tabular}{cccc}
\hline Reaction & $v=1$ & $v=2$ & $v=3$ \\
$\mathrm{CH}_{4}+\mathrm{OH}\left(v^{\prime}\right)$ & & $(2.3 \pm 0.2)[33]$ & \\
& $(0.506 \pm 0.04)[29]$ & $(2.02 \pm 0.2)[29]$ & $(4.49 \pm 0.2)[29]$ \\
& $(0.503 \pm 0.013)[34]$ & $(2.46 \pm 0.25)[34]$ & \\
& $(0.51 \pm 0.01)[27]$ & $(1.5 \pm 0.4)[27]$ & $(6.8 \pm 0.2)[27]$ \\
& $(0.21 \pm 0.09)[32]$ & & \\
$\mathrm{CO}_{2}+\mathrm{OH}\left(v^{\prime}\right)$ & $(0.218 \pm 0.05)[34]$ & $(0.686 \pm 0.075)[34]$ & \\
& & $(0.67 \pm 0.11)[33]$ & \\
$\mathrm{N}_{2} \mathrm{O}+\mathrm{OH}\left(v^{\prime}\right)$ & & $(0.46 \pm 0.06)[33]$ & \\
$\mathrm{O}_{2}+\mathrm{OH}\left(v^{\prime}\right)$ & $(0.13 \pm 0.04)[37]$ & & \\
& & $(0.26 \pm 0.04)[33]$ & \\
\hline
\end{tabular}


Table S9: Experimental initial state deactivation rate coefficients for the $\mathrm{OH}$ relaxation by effective relaxants $\mathrm{NH}_{3}, \mathrm{H}_{2} \mathrm{O}, \mathrm{CS}_{2}, \mathrm{CH}_{3} \mathrm{SH}$ and DMS. Values are given in $10^{-11} \mathrm{~cm}^{3} \mathrm{~s}^{-1}$.

\begin{tabular}{cccc}
\hline Reaction & $v=1$ & $v=2$ & $v=3$ \\
$\mathrm{NH}_{3}+\mathrm{OH}\left(v^{\prime}\right)$ & $(2.1 \pm 0.3)[26]$ & $(10.0 \pm 3.0)[26]$ & $(30.0 \pm 10.0)[26]$ \\
& $(2.86 \pm 0.12)[29]$ & $(12.6 \pm 1.2)[29]$ & \\
& $(2.49 \pm 0.22)[34]$ & $(10.1 \pm 0.9)[34]$ & \\
& & $(12.0 \pm 1.5)[33]$ & \\
$\mathrm{H}_{2} \mathrm{O}+\mathrm{OH}\left(v^{\prime}\right)$ & $(2.09 \pm 0.08)[29]$ & $(3.66 \pm 0.17)[29]$ & $(9.08 \pm 1.53)[29]$ \\
& $(1.33 \pm 0.21)[34]$ & $(2.58 \pm 0.22)[34]$ & \\
& $(1.60 \pm 0.06)[29]$ & $(2.8 \pm 0.13)[29]$ & $(6.94 \pm 1.17)[29]$ \\
$\mathrm{CS}_{2}+\mathrm{OH}(v)$ & $(1.17 \pm 0.08)[29]$ & $(1.57 \pm 0.08)[29]$ & \\
$\mathrm{DMS}+\mathrm{OH}(v)$ & $(2.55 \pm 0.99)[29]$ & $(5.34 \pm 0.73)[29]$ & \\
$\mathrm{CH}_{3} \mathrm{SH}+\mathrm{OH}\left(v^{\prime}\right)$ & $(2.51 \pm 0.13)[29]$ & $(2.60 \pm 0.21)[29]$ & \\
\hline
\end{tabular}

Table S10: Comparison of frequencies corresponding to studied molecules $\left(\mathrm{in}_{\mathrm{cm}}^{-1}\right.$ )

\begin{tabular}{cccc}
\hline $\mathrm{OH}$ & $\mathrm{SO}$ & $\mathrm{CH}_{4}$ & $\mathrm{NH}_{3}$ \\
3737.761 & 1150.791 & 2917 (Sym. stretch) & 3337 (Symmetry A1) \\
- & - & 3019 (Deg. stretch) & 3444 (Symmetry E) \\
\hline
\end{tabular}

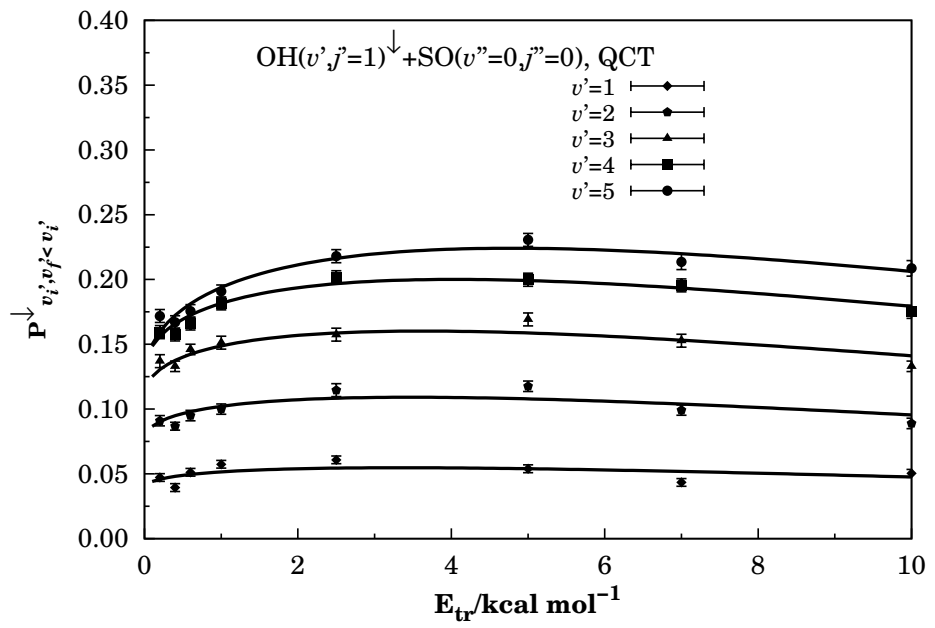

Figure S6: Specific initial-state deactivation probability for the non-reactive collisions $\mathrm{OH}+$ SO corresponding to the QCT approach. 

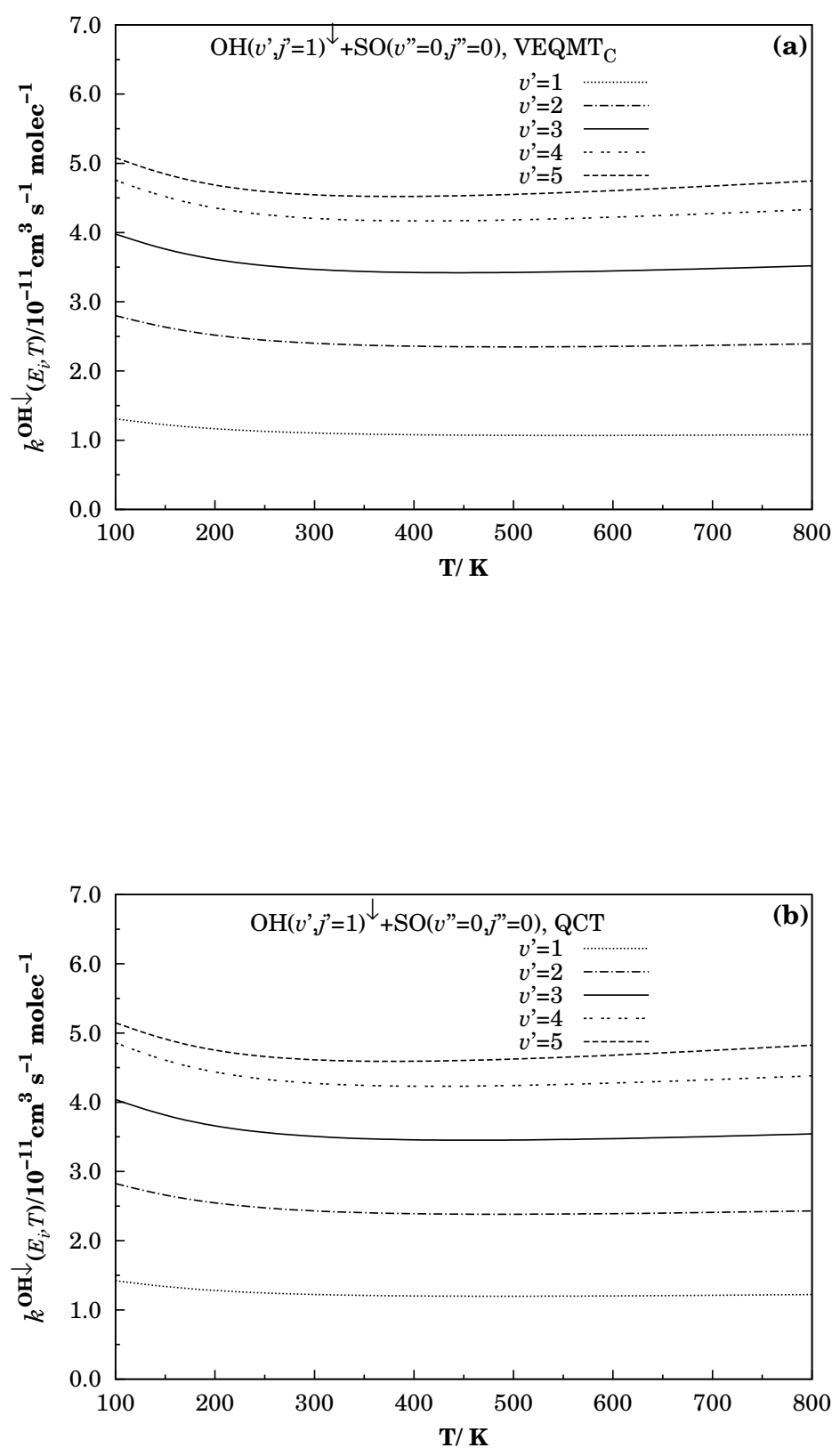

Figure S7: Specific initial-state deactivation rate coefficients for the non-reactive collisions $\mathrm{OH}+\mathrm{SO}$ corresponding to the $\mathrm{VEQMT}_{\mathrm{C}}$ (panel (a)) and QCT (panel (b)) approaches. 
Table S11: Comparison of energies corresponding to stationary states of the $\mathrm{HSO}_{2}$ system obtained in different works (in $\mathrm{kcal} \mathrm{mol}^{-1}$ ) referred to the global minimum

\begin{tabular}{|c|c|c|c|c|}
\hline $\begin{array}{c}\text { Stationary } \\
\text { state }\end{array}$ & $\begin{array}{c}\text { DMBE } \\
\text { Ballester } \\
(2005)\end{array}$ & $\begin{array}{c}\text { CASPT2(+ZPVE) } \\
\text { Garrido } \\
(2011)\end{array}$ & $\begin{array}{c}\text { CCSD }(\mathrm{T})(+\mathrm{ZPVE}) \\
\text { Rodriguez-Linares } \\
(2015)\end{array}$ & $\begin{array}{c}\operatorname{CCSD}(\mathrm{T}) \\
\text { Qin .J } \\
(2019)\end{array}$ \\
\hline HOSO & 0.0 & 0.0 & 0.0 & 0.0 \\
\hline HSO2 & 22.3 & $21.5(20.98)$ & $23.84(23.40)$ & 22.03 \\
\hline TS1 & 53.4 & $59.44(54.14)$ & $55.61(50.39)$ & 54.30 \\
\hline TS2 & 71.5 & $60.55(57.65)$ & $53.88(50.91)$ & 52.58 \\
\hline TS3 & - & $48.13(42.60)$ & $47.45(27.73)$ & 46.32 \\
\hline $\mathrm{H}+\mathrm{SO} 2$ & 44.2 & $47.81(41.63)$ & $45.56(39.44)$ & 45.10 \\
\hline $\mathrm{OH}+\mathrm{SO}$ & 71,81 & $76.01(72,50)$ & $67.17(63.66)$ & 70.59 \\
\hline
\end{tabular}

